Ann. Zootech., I970, 19 (4), 399-4II.

\title{
VARIATION DE LA SÉCRETTION DES ACIDES GRAS DES MATIERES GRASSES DU LAIT DE VACHE A LA MISE A L'HERBE \\ ET AU COURS DES SIX PREMIERES SEMAINES D'EXPLOITATION DU FOURRAGE VERT
}

\author{
C. DECAEN et M. B. GHADAKI ( $\left.{ }^{1}\right)$ \\ avec la collaboration technique de Renée Lefaivre, A. Hoden, Y. Manis et B. Marquis \\ Station de Recherches sur l'Élevage des Ruminants, \\ Centre de Recherches de Clermont-Ferrand, \\ 63 - Saint-Genès-Champanelle \\ Institut national de la Recherche agronomique
}

\section{RÉSUMÉ}

A la mise à l'herbe et au cours des six premières semaines d'exploitation du premier cycle de la plante, nous avons observé les variations de la composition en acides gras majeurs des matières grasses du lait de vache et de la quantité de ces acides gras sécrétée par jour. Seize vaches, réparties en 3 lots, ont reçu individuellement, à l'auge, du fourrage vert de luzerne, de ray-grass ou de dactyle. Une période de transition de 8 jours (graminées) ou 5 jours (légumineuses) a permis aux animaux de passer progressivement du régime hivernal (foin, ensilage, betteraves, aliment concentré) à la ration constituée exclusivement de fourrage vert.

A la mise à l'herbe, les quantités sécrétées de lait et de matières grasses et le taux butyreux augmentent. La composition des matières grasses du lait est très nettement modifiée : les proportions des acides gras courts $\left(\mathrm{C}_{4}-\mathrm{C}_{14}\right)$ et de l'acide palmitique diminuent alors que celles d'acides gras longs $\left(\mathrm{C}_{18}\right)$ augmentent, notamment celles des acides gras polyinsaturés. Ces modifications résultent d'une augmentation des quantités sécrétées d'acides gras longs $(+49$ p. IOo) qui est supérieure à la diminution (-33 p. Ioo) de celles des acides gras courts et de l'acide palmitique. Ces variations sont semblables avec chacune des trois espèces fourragères étudiées, mais l'amplitude des variations est plus marquée avec le ray-grass qu'avec la luzerne et surtout le dactyle.

Au cours des six semaines d'exploitation du premier cycle des 3 espèces fourragères, la composition en acides gras des matières grasses du lait varie peu, alors que les quantités de lait et de matières grasses sécrétées diminuent. Les vaches recevant du ray-grass maintiennent mieux leur production que celles consommant de la luzerne et surtout du dactyle.

(1) Adresse actuelle : Centre de Recherches zootechniques, Heydarabad, Karadj - Iran. 


\section{IN'TRODUCTION}

Lorsque des vaches laitières passent d'un régime alimentaire hivernal constitué essentiellement de fourrages conservés à un régime à base d'herbe verte, on observe en général des variations quantitatives et qualitatives de leur production laitière : la quantité de lait sécrétée augmente ; le taux butyreux du lait croît le plus souvent de même que la teneur du lait en matières azotées et par voie de conséquence les quantités sécrétées de matières grasses et de matières azotées augmentent nettement (cf. Demarquiliy et Journet, ig62).

A la mise à l'herbe, les caractéristiques physico-chimiques des matières grasses du lait sont également modifiées : les indices de Reichert et de saponification diminuent, l'indice d'iode augmente (Kuzdzal-Savore et Kuzdzal, I96r). Des différences se retrouvent aussi entre laits d'hiver et laits d'été (cf. Mc DowaLL, I962); elles traduisent une modification de la composition en acides gras des matières grasses : les teneurs en acides gras saturés et courts (acides gras àchaîne carbonée de I6 atomes de carbone ou moins) diminuent tandis que celles en acides gras longs et insaturés ( 18 atomes de carbone et plus) augmentent. Ces variations ont été observées dans de nombreux pays : en particulier aux Pays-Bas (STADHOUdERs et MULDER, I956), aux Etats-Unis (Jensen, Gander et SAmpugna, I962), en France (KuzdzaLSavoie et KuzDzal, I96I), en Irlande (Richardson et Mc GanN, I964), en Belgique (Guyot, I965), en Norvège (Svensen et YSTGaARD, I966), en Finlande (ANTILLA, I966), en U. R. S. S. (ARTamentova et Martynushrina, I966). Ces différences saisonnières sont expliquées le plus souvent par des considérations alimentaires (cf. MATTSSON, I962), les rations estivales, constituées d'herbe verte, étant plus riches en acides gras longs insaturés particulièrement en acide linolénique que les rations hivernales à base de foin et d'ensilage.

Si de nombreuses études traitent des variations saisonnières de la composition en acides gras des matières grasses du lait, peu décrivent l'influence spécifique de la mise à l'herbe (HAwkE, I963; KUzDzAL-SAvoIe, I964) et rares sont les chercheurs qui ont mesuré les variations de la quantité d'acides gras sécrétée.

Par ailleurs, peu d'auteurs ont essayé, comme Mc Dowal, et Mc Gillivray (I963), d'observer l'influence de la nature de l'herbe consommée et de son stade de végétation. C'est pourquoi l'objectif de cette étude a été de décrire l'évolution qualitative et quantitative de la sécrétion des acides gras des triglycérides du lait, au moment de la mise à l'herbe et au cours de l'exploitation du premier cycle végétatif de trois espèces fourragères différentes : luzerne, ray-grass et dactyle. Ce travail fait partie d'une thèse de docteur-ingénieur soutenue par l'un d'entre nous (GHADAKI, Ig68).

\section{MATÉRIEI, E'T MÉTHODES}

\section{Animaux - Alimentation}

Nous avons observé la production laitière de I6 vaches de race Pie Noive, Pie Rouge ou Normande qui étaient en Ire ou 2 e lactation. Au début de l'expérience (en avril r965), ces animaux avaient mis bas depuis 4 à 5 mois et leur niveau de production laitière était assez faible (9 à $14 \mathrm{~kg}$ de lait/jour) ; nous les avons choisis ainsi pour ne pas avoir à leur distribuer d'aliments concentrés. 
Durant les 3 mois qui ont précédé l'expérience, les vaches avaient consommé du foin, de l'ensilage et un aliment concentré. En cours d'expérience, deux lots de 5 à 6 vaches ont reçu, à l'auge, respectivement de la luzerne et du ray-grass d'Italie (Rina) à l'étable de la Minière et un lot de 5 vaches a été alimenté avec du dactyle à Jouy. Au cours d'une période de transition de 8 jours pour les graminées et de 15 jours pour la luzerne, les vaches ont reçu des quantités croissantes de fourrage vert et des quantités décroissantes de fourrages conservés et de concentré. L'alimentation exclusive au fourrage vert a commencé le i i avril pour le ray-grass, le 26 pour le dactyle et le I er mai pour la luzerne; nos observations se sont poursuivies au cours des 6 premières semaines d'exploitation du premier cycle végétatif de ces plantes. Les vaches ont reçu en 4 repas quotidiens $(5 \mathrm{~h} 30,9 \mathrm{~h}$ 3o, $\mathrm{I} 3 \mathrm{~h} 30$ et $\mathrm{I} 7 \mathrm{~h}$ ) le fourrage vert qu'elles ont consommé à volonté (Io p. Ioo de refus au moins). Une vache produisant plus de $16 \mathrm{~kg}$ de lait par jour a reçu $0,5 \mathrm{~kg}$ d'aliment concentré par $\mathrm{kg}$ de lait produit au-delà de cette quantité.

\section{Mesures effectuées - Présentation des résultats}

Nous avons mesuré les quantités de matière sèche de fourrage vert ingérées par chaque vache, par pesée journalière des quantités offertes et refusées et détermination de la teneur en matière sèche de ce fourrage vert à chaque distribution. Les caractéristiques morphologiques de ces fourrages verts et leurs coefficients d'utilisation digestive ont été mesurés sur des moutons (DEMARQUILLY) ; ces données sont rapportées dans le tableau I.

Nous avons mesuré chaque jour, pour chaque vache, la quantité de lait produite, le taux butyreux sur un échantillon représentatif des deux traites et la quantité de matières grasses.

Sur un échantillon représentatif des 4 traites consécutives de chaque mardi et mercredi, nous avons déterminé la composition en acides gras des matières grasses du lait, selon la méthodologie décrite par DECAEN et ADDA (I966), par chromatographie en phase gazeuse des esters méthyliques et des esters butyliques des acides gras; nous avons, de plus, estimé les quantités sécrétées des différents acides gras. Utilisant un détecteur à catharomètre, nous avons exprimé nos résultats en proportions pondérales d'esters méthyliques pour la composition des matières grasses et en grammes d'esters méthyliques pour les quantités sécrétées. Pour présenter plus synthétiquement nos observations, nous avons classé les acides gras en 3 groupes : acides gras courts, acide palmitique et acides gras longs.

\section{RÉSULTATS}

\section{Infuence de la mise à l'herbe}

Entre la dernière semaine de régime de type hivernal et la première semaine d'alimentation exclusive au fourrage vert (fig. I, tab1. 2), les quantités sécrétées de lait et de matières grasses augmentent $(P<0,05)$ avec le ray-grass $(+7,3$ et $+20,0$ p. I0o) et la luzerne $(+8$ et $+7,9$ p. I0o $)$, mais diminuent $(P<0,05)$ avec le dactyle ( - II et $-6,2 \mathrm{p}$. I00) ; le taux butyreux du lait augmente $(\mathrm{P}<0,05)$ davantage avec le ray-grass $(+4,3 \mathrm{~g} / \mathrm{kg} ; \mathrm{P}<0,05)$ qu'avec le dactyle $(+2,2 \mathrm{~g} / \mathrm{kg}$; $\mathrm{P}>0,05)$ et surtout la luzerne $(0,5 \mathrm{~g} / \mathrm{kg} ; \mathrm{P}>0,05)$.

$\mathrm{I}_{\text {a }}$ composition en acides gras des matières grasses du lait varie très nettement au cours de cette même période : les proportions d'acides gras longs (plus particulièrement des acides insaturés) augmentent $(P<0,005)$ au détriment de celles d'acides gras courts $(\mathrm{P}>0,020)$ et d'acide palmitique $(\mathrm{P}<0,00 \mathrm{I})$ (fig. 2). On observe des modifications semblables pour chacune des 3 espèces fourragères, mais l'amplitude des variations est plus importante avec le ray-grass qu'avec le dactyle ou la luzerne, notamment en ce qui concerne les acides gras longs (tabl. 2, fig. I).

Le changement de régime entraîne une augmentation des quantités sécrétées d'acides gras longs $(+49 \mathrm{p}$. Ioo) et une diminution de celles des acides gras courts ( - I6 p. I00) et de 1'acide palmitique (- I7 p. I0o). La sécrétion des acides gras 


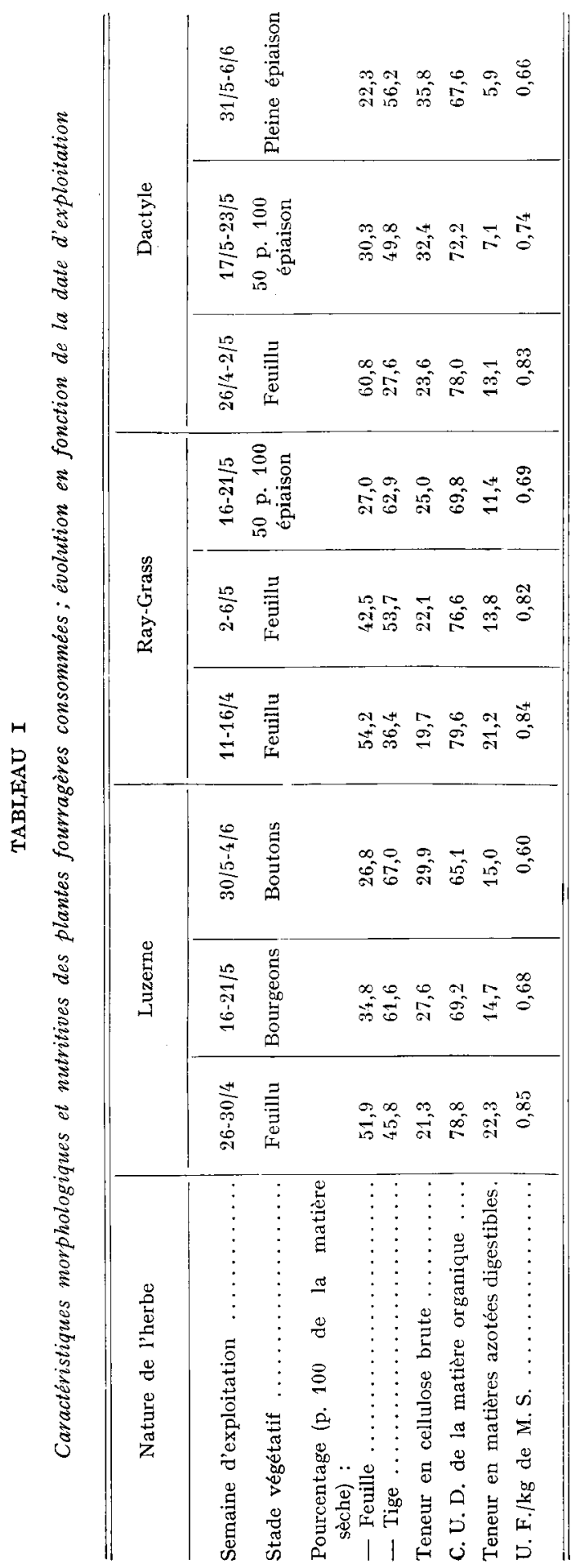


courts $\left(\mathrm{C}_{4}\right.$ à $\left.\mathrm{C}_{12}\right)$ a tendance à diminuer d'autant plus que leur chaîne carbonée est plus longue (fig. I); la sécrétion des acides myristique $\left(\mathrm{C}_{14}\right)$ et palmitique $\left(\mathrm{C}_{16}\right)$ est presque deux fois moins affectée (en valeurs relatives) que celle de l'acide laurique $\left(C_{12}\right)$. Les quantités d'acide oléique augmentent légèrement plus que celles d'acide stéarique, mais moins que celles d'acides diènes et triènes. Avec le ray-grass, la quan-

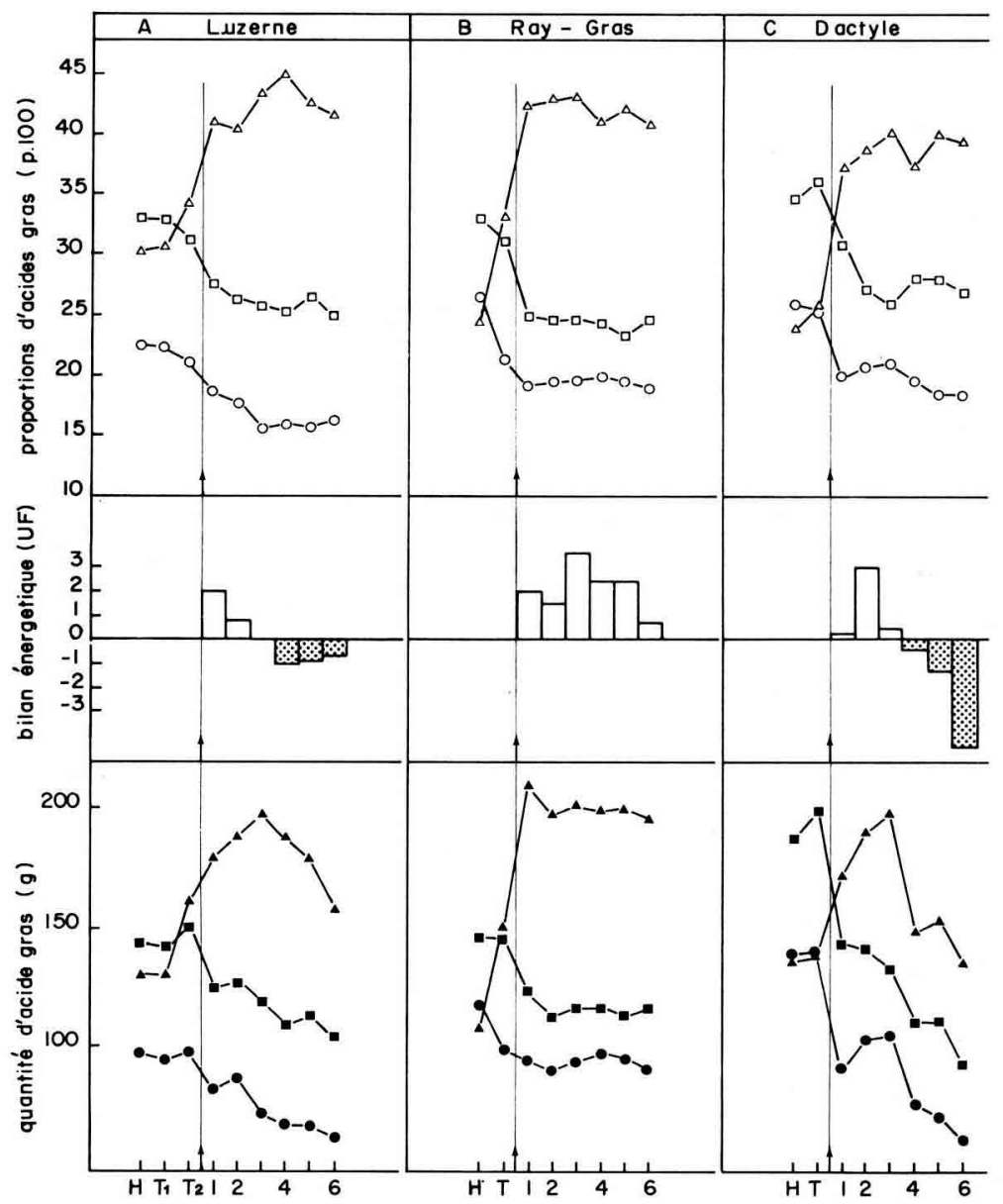

FIG. I. - Variations de la sécrétion des acides gras à la mise à l'herbe et au cours du premier cycle de végétation de la luzerne, du ray-grass et du dactyle

$\mathrm{H}$ : régime hivernal

$\mathrm{T}$ : régime de transition

I, $2 \ldots, 6:$ : semaines d'exploitation

Acides gras courts : proportions o-—o; quantités

Acide palmitique : proportions $\square-\square$; quantités

Acides gras longs : proportions $\Delta \longrightarrow \Delta$; quantités

tité sécrétée d'acides gras longs double presque, alors qu'elle n'augmente que d'un tiers environ avec la luzerne et le dactyle (tabl. 2, fig. 2) ; avec le dactyle, la sécrétion d'acides gras courts et d'acide palmitique diminue beaucoup plus nettement qu'avec les 2 autres espèces fourragères. Au cours de la période de transition alimentaire, on 
TABLEAU 2

Variations relatives de la sécrétion des acides gras des glycérides du lait entre la dernière semaine de régime hivernal et la première semaine de régime exclusif à l'herbe

\begin{tabular}{|c|c|c|c|c|c|c|}
\hline & \multicolumn{3}{|c|}{ Proportions } & \multicolumn{3}{|c|}{ Quantités } \\
\hline & $\begin{array}{c}\text { Acides } \\
\text { gras courts }\end{array}$ & $\begin{array}{c}\text { Acide } \\
\text { palmitique }\end{array}$ & $\begin{array}{l}\text { Acides } \\
\text { gras longs }\end{array}$ & $\begin{array}{c}\text { Acides } \\
\text { gras courts }\end{array}$ & $\begin{array}{c}\text { Acide } \\
\text { palmitique }\end{array}$ & $\begin{array}{l}\text { Acides } \\
\text { gras longs }\end{array}$ \\
\hline Lot A : Luzerne... & -17 & -17 & $+3 \dot{t}$ & -15 & -13 & +37 \\
\hline Lot B : Ray-grass. & -25 & -27 & -f $7 \prime_{t}^{\prime}$ & -19 & -15 & +93 \\
\hline Lot C : Dactyle... & -11 & -22 & +55 & -34 & -23 & +26 \\
\hline Ensemble ....... & -21 & -18 & +50 & -16 & -17 & +49 \\
\hline
\end{tabular}
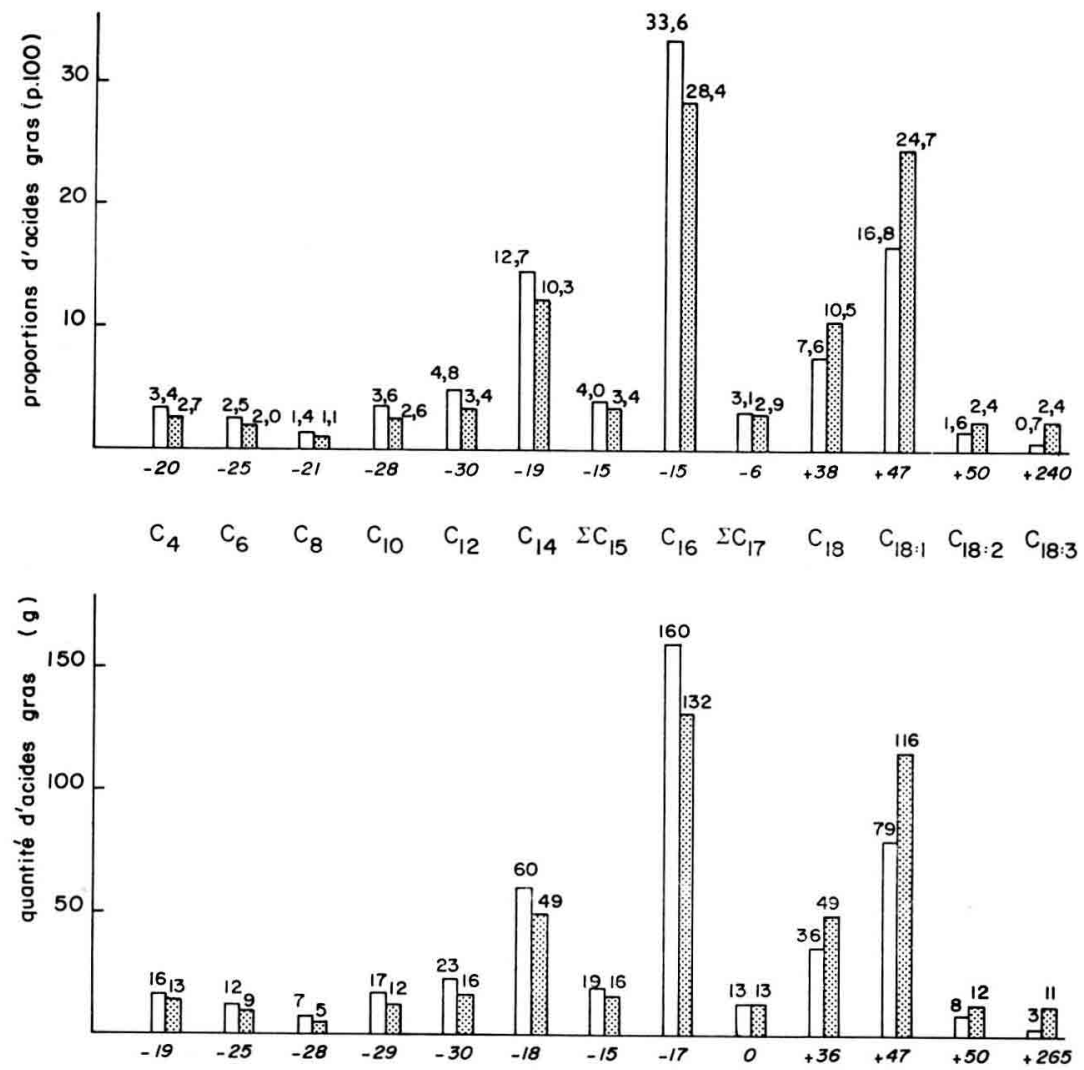

FIG. 2. - Variations de la sécrétion des acides gras des triglycérides du lait à la mise à l'herbe

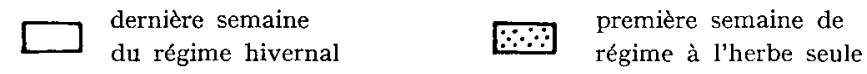

Le nombre au-dessus indique la proportion (p. Ioo) ou la quantité (g).

Le nombre au-dessous (axe des $\mathrm{x}$ ) précise la variation relative en p. Ioo. 
observe des modifications de la sécrétion du lait et des matières grasses, dès que l'on distribue du fourrage vert. Ces modifications s'accentuent au fur et à mesure que l'on distribue des quantités croissantes de fourrage vert. On observe, avec le ray-grass, une évolution plus précoce et plus rapide qu'avec la luzerne ou le dactyle.

\section{Influence du stade de végétation de l'herbe}

Au cours des 6 premières semaines du premier cycle d'exploitation du fourrage vert, en moyenne pour les 3 espèces fourragères, le taux butyreux (tabl. 3) et la composition en acides gras des matières grasses du lait (fig. I) varient peu, alors que les quantités sécrétées de lait et de matières grasses diminuent respectivement de

TABLEAU 3

Variations à la mise à l'herbe et au cours des 6 premières semaines de végétation des paramètres de consommation et de production laitière sur luzerne, ray-grass et dactyle

\begin{tabular}{|c|c|c|c|c|c|c|c|c|c|}
\hline & & \multirow{2}{*}{$\begin{array}{l}\text { Régime } \\
\text { hivernal }\end{array}$} & \multirow{2}{*}{ Transition } & \multicolumn{6}{|c|}{$\begin{array}{c}\text { Stade de végétation de l'herbe } \\
\text { (semaines) }\end{array}$} \\
\hline & & & & 1 & 2 & 3 & 4 & 5 & 6 \\
\hline $\begin{array}{l}\text { Quantité d'herbe } \\
\text { ingérée (kg de } \\
\text { matière sèche) }\end{array}$ & $\begin{array}{l}\text { Luzerne } \\
\text { Ray-grass } \\
\text { Dactyle }\end{array}$ & & & $\begin{array}{l}13,1 \\
13,6 \\
11,7\end{array}$ & $\begin{array}{l}12,9 \\
12,6 \\
14,0\end{array}$ & $\begin{array}{l}12,1 \\
1 / 4,4 \\
12,1\end{array}$ & $\begin{array}{r}11,3 \\
14,1 \\
9,6\end{array}$ & $\begin{array}{l}12,1 \\
14,7 \\
10,4\end{array}$ & $\begin{array}{r}12,1 \\
14,1 \\
5,3\end{array}$ \\
\hline $\begin{array}{c}\text { Quantité de matière } \\
\text { organique digestible } \\
\text { ingérée }(\mathrm{kg})\end{array}$ & $\begin{array}{l}\text { Luzerne } \\
\text { Ray-grass } \\
\text { Dactyle }\end{array}$ & & & $\begin{array}{l}9,1 \\
9,2 \\
7,9\end{array}$ & $\begin{array}{l}8,4 \\
8,7 \\
9,8\end{array}$ & $\begin{array}{r}7,8 \\
10,2 \\
8,1\end{array}$ & $\begin{array}{l}7,0 \\
9,6 \\
6,2\end{array}$ & $\begin{array}{l}7,3 \\
9,8 \\
6,2\end{array}$ & $\begin{array}{l}7,1 \\
8,8 \\
3,2\end{array}$ \\
\hline $\begin{array}{l}\text { Bilan } \\
\text { énergétique } \\
(\mathrm{U} . \mathrm{F} .)\end{array}$ & $\begin{array}{l}\text { Luzerne } \\
\text { Ray-grass } \\
\text { Dactyle }\end{array}$ & & & $\begin{array}{l}+2,0 \\
+2,0 \\
+0,3\end{array}$ & $\begin{array}{r}+0,8 \\
+1,5 \\
+3,0\end{array}$ & $\begin{array}{l}-0,1 \\
+3,6 \\
+0,5\end{array}$ & $\begin{array}{r}-1,0 \\
+2,4 \\
-0,5\end{array}$ & $\begin{array}{r}-0,9 \\
+2,4 \\
-1,3\end{array}$ & $\begin{array}{r}-0,7 \\
+0,7 \\
-4,5\end{array}$ \\
\hline $\begin{array}{l}\text { Quantité } \\
\text { de lait } \\
(\mathrm{kg})\end{array}$ & $\begin{array}{l}\text { Luzerne } \\
\text { Ray-grass } \\
\text { Dactyle }\end{array}$ & $\begin{array}{l}12,3 \\
12,4 \\
1 ' t, 9\end{array}$ & $\begin{array}{l}13,0 \\
11,9 \\
14,0\end{array}$ & $\begin{array}{l}13,3 \\
13,3 \\
13,3\end{array}$ & $\begin{array}{l}12,9 \\
12,5 \\
11,8\end{array}$ & $\begin{array}{l}12,4 \\
12,9 \\
12,2\end{array}$ & $\begin{array}{l}12,0 \\
12,4 \\
11,5\end{array}$ & $\begin{array}{r}11,6 \\
12,0 \\
9,6\end{array}$ & $\begin{array}{r}11,2 \\
11,6 \\
9,9\end{array}$ \\
\hline $\begin{array}{c}\text { Taux butyreux } \\
\text { du lait } \\
(\mathrm{g} / \mathrm{kg})\end{array}$ & $\begin{array}{l}\text { Luzerne } \\
\text { Ray-grass } \\
\text { Dactyle }\end{array}$ & $\begin{array}{l}34,6 \\
34,8 \\
37,5\end{array}$ & $\begin{array}{l}35,8 \\
38,1 \\
37,5\end{array}$ & $\begin{array}{l}35,1 \\
39,1 \\
38,5\end{array}$ & $\begin{array}{l}35,5 \\
38,8 \\
39,7\end{array}$ & $\begin{array}{l}37,5 \\
37,4 \\
41,5\end{array}$ & $\begin{array}{l}36,7 \\
40,1 \\
40,2\end{array}$ & $\begin{array}{l}37,3 \\
40,4 \\
39,5\end{array}$ & $\begin{array}{l}36,0 \\
41,7 \\
37,5\end{array}$ \\
\hline $\begin{array}{c}\text { Quantité } \\
\text { de matières grasses } \\
(\mathrm{g})\end{array}$ & $\begin{array}{l}\text { Luzerne } \\
\text { Ray-grass } \\
\text { Dactyle }\end{array}$ & $\begin{array}{l}428 \\
431 \\
557\end{array}$ & $\begin{array}{l}466 \\
455 \\
538\end{array}$ & $\begin{array}{l}493 \\
518 \\
528\end{array}$ & $\begin{array}{l}455 \\
487 \\
492\end{array}$ & $\begin{array}{l}459 \\
484 \\
502\end{array}$ & $\begin{array}{l}432 \\
494 \\
454\end{array}$ & $\begin{array}{l}421 \\
481 \\
384\end{array}$ & $\begin{array}{l}398 \\
480 \\
373\end{array}$ \\
\hline
\end{tabular}

I 8 à I 4,5 p. Ioo. Pendant ce temps, la valeur nutritive de l'herbe a diminué (tabl. I) : en moyenne le coefficient d'utilisation digestive de la matière organique est passé de 78,8 à 57,5 p. IOO $(0,83$ à 0,65 U. F.) et les teneurs en matières azotées digestibles de la matière sèche de 18,9 à I0,8 p. I00. Les quantités de matière organique digestible ingérées par les vaches ont diminué de $-27 \mathrm{p}$. Ioo. Cependant ces phénomènes varient d'amplitude selon la nature du fourrage vert ingéré (tab1. 3, fig. I).

I a luzerne, pendant les 3 premières semaines d'exploitation, jusqu'au stade du bourgeonnement, est bien consommée (plus de $8 \mathrm{~kg}$ de matière organique digestible 
ingérée) et permet à la vache d'assurer des bilans énergétiques et azotés positifs ; mais au cours des 3 semaines qui suivent (jusqu'à l'apparition des boutons floraux), les quantités ingérées diminuent $(7,0 \mathrm{~kg}$ de $\mathrm{M}$. O. D.) et le bilan énergétique devient négatif. Pendant ce temps, les quantités de lait et de matières grasses produites diminuent respectivement de I5 et 19 p. Ioo en 6 semaines. Jusqu'au bourgeonnement, le taux butyreux augmente légèrement tandis que les quantités sécrétées d'acides gras longs augmentent et que celles d'acides gras courts et d'acide palmitique diminuent. Ensuite, jusqu'à l'apparition des boutons floraux, la proportion d'acides gras longs diminue et les quantités d'acides gras longs décroissent plus rapidement que celles des acides gras courts et de l'acide palmitique.

Le ray-grass, parmi les 3 espèces étudiées, est le fourrage vert le mieux consommé : au cours des 6 semaines d'exploitation du stade feuillu au début de l'épiaison, les quantités de matière organique digestible ingérées par les vaches restent élevées (9 à $10 \mathrm{~kg}$ ) et les bilans énergétique et azoté des animaux positifs. Les quantités de lait et de matières grasses diminuent assez lentement respectivement de 12 et 7 p. IOo en 6 semaines; le taux butyreux a tendance à augmenter alors que la composition en acides gras des matières grasses du lait reste stable.

Le dactyle, au cours des 6 semaines d'observation passe du stade feuillu à la pleine épiaison. Bien que le coefficient de digestibilité de la matière organique ne diminue pas plus que celui de la luzerne, les quantités ingérées de matière organique baissent très nettement à partir de la $4^{\mathrm{e}}$ semaine et atteignent un niveau très bas en fin d'expérience $(5,3 \mathrm{~kg}$ de matière organique digestible); les animaux sont sousalimentés en énergie et en azote au cours des 3 dernières semaines. Les quantités de lait diminuent davantage (-25 p. Ioo) qu'avec le ray-grass et la luzerne, de même que les quantités de matières grasses (-29 p. IOO), surtout au cours des 3 dernières semaines ; cependant, la composition en acides gras des matières grasses varie peu et les quantités sécrétées de chacun des acides gras se maintiennent à leur niveau initial ou augmentent durant les 3 premières semaines, mais ensuite elles décroissent très rapidement.

\section{Variations individuelles}

Il est difficile de tirer des conclusions à la lecture des coefficients de corrélation entre les paramètres de production laitière et de composition des matières grasses du lait, rapportés dans le tableau 4, car l'effectif ( 16 données) est faible. Cependant, on peut noter que les liaisons entre les paramètres étudiés sont différentes sur régime hivernal et sur régime à l'herbe.

Dans les deux cas, la quantité de matières grasses sécrétée est fortement et positivement liée à la production laitière. Avec le régime hivernal, elle varie dans le même sens que le taux butyreux et que la proportion d'acide palmitique, mais indépendamment des proportions d'acides gras longs et de celles des acides gras courts. Cependant, avec l'alimentation à l'herbe, elle varie de manière indépendante du taux butyreux, dans le même sens que les proportions d'acides gras courts et d'acide palmitique et en sens contraire des porportions d'acides gras longs. D'une manière générale, le taux butyreux du lait varie, entre individus, en sens contraire de la proportion d'acides gras longs insaturés dans les matières grasses du lait ; avec le régime hivernal, il varie dans le même sens que la proportion d'acide palmitique. 

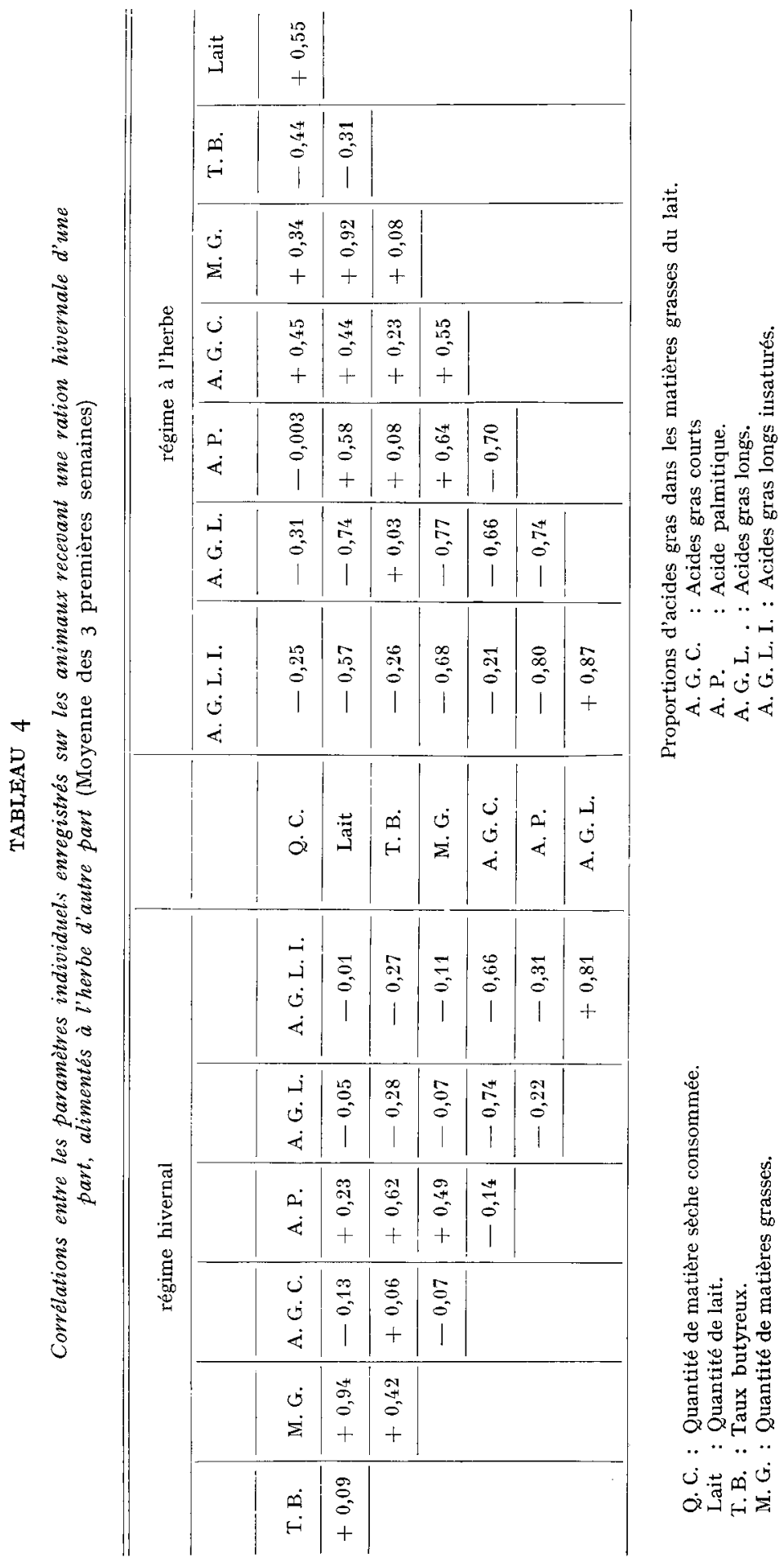
Les vaches qui consomment le plus d'herbe, sont celles qui produisent le plus de lait et de matières grasses, mais leur lait aurait un faible taux butyreux et des matières grasses relativement riches en acides gras courts et pauvres en acides gras longs.

\section{DISCUSSION}

Nos observations confirment qu'à la mise à l'herbe, d'une part, les quantités de lait et de matières grasses produites par la vache, ainsi que le taux butyreux, augmentent (DEMARQUILIY et JOURNET, I962), d'autre part, que la composition en acides gras des matières grasses du lait est modifiée : les proportions des acides gras longs, plus particulièrement des insaturés, augmentent (KuzDzaL-SAvorE, I964).

Nous montrons que l'augmentation de la sécrétion des matières grasses et les modifications de leur composition, à la mise à l'herbe, sont dues à une augmentation des quantités d'acides gras longs sécrétées qui est largement supérieure à la diminution des quantités sécrétées d'acides gras courts et d'acide palmitique. L'amplitude de variation de ces phénomènes dépend de l'espèce fourragère consommée, les vaches qui reçoivent du ray-grass accroissent davantage leur production de lait et de matières grasses que celles consommant de la luzerne et surtout du dactyle. Ces dernières observations sont en accord avec les résultats de DEMARQUILLY (Ig63).

Ces modifications de la sécrétion des acides gras des matières grasses du lait à la mise à l'herbe peuvent être reliées à certaines caractéristiques nutritives du fourrage vert : aliment très digestible dont les lipides constitutifs sont riches en acides gras longs insaturés. Bien que n'ayant pas déterminé les teneurs en lipides des fourrages verts consommés, on peut estimer qu'ils contenaient, dans leur matière sèche, 5 à 8 p. Ioo de lipides riches en acides gras longs insaturés, notamment en acide linolénique : Garton (I960), Shorland (I96I), Hawke (I963), Kuzdzal-Savoie (I964), CzERKAwski (I967) ; ces acides gras insaturés sont dans le rumen partiellement hydrogénés (Shorland, WEFnink et Johns, I955; WRight, I959; Viviani et Lenaz, I963; WARd, ScotT et DAwson, I964) et isomérisés (ShorLand, WeENINK et Johns, I955, I957). La mise à l'herbe provoque un apport accru d'acides gras longs, notamment d'acide oléique au niveau de l'intestin et du tissu mammaire, ce qui expliquerait l'augmentation des quantités sécrétées des acides gras longs dans les matières grasses du lait (STORRy, TUCkEy et HAI, I, I969 ; STEELE, I969; Noble, SteEle et MoOrE, I969 ; MoOre, SteEle et Noble, I969). Cependant, le plus souvent lorsqu'on ajoute à la ration des suppléments d'acides gras insaturés, on observe une chute du taux butyreux et non une augmentation, d'autant plus que les apports sont plus importants et les graisses plus insaturées : huile de maïs, de soja..., ces mêmes graisses ou huiles, après avoir été hydrogénées n'ont plus le même effet (cf. revue de KIRCHGESSNER, FrieseckE et КоCH, I967). Cependant des injections intraveineuses d'huile de soja, bien que riche en acide linoléique, élèvent le taux butyreux du lait et la quantité de matières grasses sécrétées (STORRY et al., I969). Donc, l'influence de la mise à l'herbe viendrait de la richesse de l'herbe en acide linolénique, acide gras insaturé facilement hydrogéné dans le rumen (HAWkE et RoBERTSON, I964 ; ROBERTson et HAWKE, I964; BATH et HILL, I964). L'apport en grande quantité des acides gras longs pourrait, en partie, expliquer les faibles proportions d'acides gras courts et d'acide palmitique dans les matières grasses du lait; en effet, les acides gras longs, 
lorsqu'ils sont présents en grande quantité seraient utilisés préférentiellement par le tissu mammaire pour former les triglycérides du lait.

Par ailleurs, le fourrage vert, surtout à l'état jeune, est pauvre en constituants membranaires et très digestible (JARRIGE et Minson, I964). A la mise à l'herbe, on observe des modifications des fermentations digestives au niveau du rumen; la production totale d'acides gras volatils augmente et la proportion d'acide acétique dans le mélange d'acides gras volatils diminue alors que celles d'acides propionique et butyrique augmentent (JoHNs, I955 ; BALCH et RowLAND, I957 ; ANNISON et al., I 959 ; BATH et Rook, I96I ; BATH et al., I962 ; BATH et RooK, I965). Ces modifications digestives pourraient, elles aussi, expliquer en partie la baisse de sécrétion des acides gras courts et palmitique. Toutefois, leur effet est peut-être assez faible car la quantité totale d'acide acétique produite dans le rumen ne diminue pas.

Au cours des 6 semaines d'exploitation du premier cycle de végétation du fourrage vert, nous n'observons pas une variation très nette de la composition des matières grasses du lait, notamment avec le ray-grass. Ces résultats diffèrent de ceux de HAWKE (I963), qui observe une influence importante du stade de végétation đu raygrass sur la composition des matières grasses du lait : avec le fourrage vert, au stade jeune ( 7 à $\mathrm{Io} \mathrm{cm}$ ), les matières grasses du lait sont plus riches en acide butyrique et en acides oléique et stéarique et plus pauvres en acides myristique et palmitique qu'avec le fourrage vert consommé à un stade avancé ( $30 \mathrm{~cm}$ environ). Ces différences entre nos résultats et ceux de HAwKe (I963) tiennent peut-être à ce que nous avons étudié un ray-grass de variété différente (Rina italien) et exploité à des stades végétatifs plus avancés. Le bon maintien de la sécrétion des acides gras des matières grasses du lait, au cours de l'exploitation du ray-grass, semble lié en ce qui nous concerne, à des niveaux d'ingestion qui restent élevés, entraînant le maintien des bilans énergétique et azoté positifs ; il n'en est pas de même avec la luzerne et le dactyle. Avec ces deux espèces fourragères, tant que les niveaux d'ingestion et la valeur nutritive du fourrage vert permettent à la vache d'assurer son équilibre énergétique et azoté, les quantités sécrétées se maintiennent bien et les matières grasses ont tendance à s'enrichir en acides gras longs : il en est ainsi jusqu'au bourgeonnement de la luzerne et jusqu'au début de l'épiaison du dactyle; en revanche, par la suite, la qualité du fourrage vert est insuffisante et les vaches sont sous-alimentées : les quantités sécrétées diminuent mais la composition des matières grasses varie peu. Ainsi avec du fourrage vert, une sous-alimentation progressive (de Io p. Ioo avec la luzerne, de $5^{\circ} \mathrm{p}$. Ioo avec le dactyle) ne provoque pas les mêmes modifications de la composition des matières grasses sécrétées qu'une sous-alimentation brutale de 50 p. Ioo avec un régime hivernal (DECAEN et ADDA, I970) : en effet, dans le cas présent, la réduction des apports alimentaires provoquée par l'avancement du stade végétatif du dactyle consommé entraîne une diminution de la sécrétion des acides gras longs alors que la réduction brutale de $5^{\circ} \mathrm{p}$. Ioo des apports alimentaires d'une ration hivernale augmentait de 50 p. Ioo la sécrétion des acides gras longs. Cette différence de réaction est peut-être due à la nature de la ration, mais aussi au fait que, dans le cas de l'alimentation à l'herbe verte, la sous-alimentation est progressive et l'animal peut s'adapter sans subir de stress. 


\section{SUMMARY}

\section{FATTY ACID SECRETION IN THE MILK FAT OF GREEN FORAGE FED COWS EFFECTS OF CHANGING FROM WINTER FEEDING TO ZERO GRAZING AND OF THE FORAGE GROWTH STAGE}

The influence of changing from winter feeding to zero grazing and that of the green forage growth stage (Ist cycle) upon

a) the fatty acid composition of milk fat

b) the daily secretion of these fatty acids were studied during the first six weeks of crop.

I 6 cows, receiving hay, silage and concentrate before the trial, were distributed into three lots. After one or two weeks of feeding transition, the animals of each lot were individually stall fed with green forage : either with lucerne, cocksfoot or ray-grass.

Changing from winter feeding to zero grazing resulted in an increase of the butterfat percentage and, except for cocksfoot, in a higher production of milk and milk fat (table 3 ). It also induced changes in the fatty acid composition of the milk fat (fig. I, table 2) : the proportions of short fatty acids $\left(\mathrm{C}_{4}-\mathrm{C}_{14}\right)$ and of palmitic acid decreased, and those of the long fatty acids $\left(\mathrm{C}_{18}\right)$, especially poly-unsaturated acids, increased. These changes are due to an increase of the secretion of long fatty acids, which is higher than the decrease of the palmitic and short fatty acid secretion.

During the first six weeks of green forage crop, as the nutritive value of the plant diminishes (table $\mathbf{1}$ ), we noticed :

- only a small variation in the fatty acid composition of the milk fat ; centage.

- a decrease of the milk and butterfat secretion and a slight increase of the butterfat per-

These effects were more striking in ray-grass fed cows than in those receiving lucerne and particularly cocksfoot.

\section{RÉFÉRENCES BIBIIOGRAPHIQUES}

Annison E. F., Lewis D., Linsay D. B., I959. The metabolic changes which occur in sheep transferred to lush springgrass. I. Changes in blood and rumen constituents. J. A gric. Sci., 53, 34-4I.

Antilla V., I966. Fatty acid composition, solidification and melting points of finnish butterfat. Meyeritiet Aikakausk., 27 (1), 72 p.

Artamentova V., Martynushikina A., I 966 . Estimation of the composition of butterfat by gas chromatography. Moloch. Prom., $2^{\prime y}$ (6), I7-1 8 in D. S. A. 325 I, 28, (10).

BALCH D. A., Rowland S. J., 1957. Volatile fatty acids and lactic acid in the rumen of dairy cows receiving a variety of diets. Brit. J. Nutr., 11, 288-298.

Bath I. H., Rook J. A. F., I96r. The effects of stage of growth of $S 23$ perennial rye-grass on the production of volatile fatty acids in the rumen of the cow. Proc. Nutr. Soc, 20, XV. (Abstr.).

Bath I. H., Rook J. A. F., Rowland S. J., rg62. Effects of grazing on the ruminal production of volatile fatty acids in the relation to the protein and fat contents of milk fat. XVI Congr. Intern. de Laiterie. Sect. A., 49-56.

Bath I. H., Rook J. A. F., 1965. The evaluation of cattle foods and diets in terms of ruminal concentration of volatile fatty acids. II. Roughage and succulents. J. Agric. Sci., 64, 67-75.

Bath I. H., Hill K. J., I965. Ruminal lipolysis and hydrogenation of lipids in the digestive tract of the sheep. Proc. Nutr. Soc., London Dec. I964, 24, XII-XIII.

Czerkawski J. W., 1967. The effect of storage on the fatty acid of dried rye-grass. Br. J. Nutr., 21, $599-608$.

DecaEN C., ADDA J., I970. Influence d'une sous-alimentation passagère sur la sécrétion des principaux acides gras du lait et sur les concentrations en acides-gras-non-estérifiés et en triglycérides du sang chez la vache. Ann. Biol. anim. Bioch. Biophys., (sous presse).

Demarquilly C., Journet M, I962. Variation de la composition du lait à la mise à l'herbe. $X V I^{\mathrm{e}}$ Congr. Intern. de Laiterie., Section A, I, 33-48.

Demarquilly C., I963. Influence de la nature du pâturage sur la production laitière et la composition du lait. Ann. Zootech., 12, 69-I04. 
Mc Dowall F. H., I962. Studies on the properties of New Zealand butterfat. V. The effect of stage of lactation. J. Dairy Res., 29, 307-320.

Mc Dowall F. H., McGillivray W. A., 1963. Studies on the properties of New Zealand butterfat. VI. Comparison of the properties and vitamin A potencies of butterfats produced by clover-fed and ryegrass-fed cows. J. Dairy Res., 30, 47-57.

GHADAKI M. B., I968. Influence de quelques facteurs alimentaires sur la sécrétion des principaux acides gras du lait de vache. Thèse de Docteur Ingénieur de l'Université de Clermont-Ferrand, I4I $\mathrm{p}$.

Guyor A., I965. Composition de la matière grasse des beurres belges et recherches des falsifications par chromatographie en phase gazeuse. J. Pharm. de Belg., 9-10, 367-396.

HAwke J. C., I963. Studies on the properties of New Zealand butterfat. VII. The fatty acid composition of the milk fat of cow grazing on rye-grass at two stages of maturity and the composition of the rye-grass lipids. J. Dairy Res., 30, 67-75.

Hawke J. C., Robertson J. A., I964. Studies on rumen metabolism. II. In vivo hydrolysis and hydrogenation of lipid. J. Sci. Fd Agric., 15, 283-289.

Jarrige R., Minson D. J., I964. Digestibilité des constituants du ray-grasse anglais $S 24$ et du dactyle $S$ 37, plus spécialement des constituants glucidiques. Ann. Zootech., 14, I I7-I50.

Jensen R. G., Gander G. W., Sampugna J., r962. Fatty acid composition of the lipids from pooled raw milk. J. Dairy Sci., 45, 329-33I.

JoHns A. T., 1965. Pasture quality and rumen digestion. 2. Level of volatile acids and ammonia in the rumen of sheep on a high production pasture. N.Z.J. Sci. Technol. (A), 37, 323-33I.

Kirchgessner M., Friesecke H., Koch G., r967. Nutrition and the composition of milk. Crosby Lockwood and Son Ltd. Londres, $273 \mathrm{p}$.

Kuzdzal-Savoie S., Kuzdzal W., r96r. Influence de la mise à l'herbe des vaches laitières sur les indices de la matière grasse du beurre et sur les teneurs en différents acides gras polyinsaturés. Ann. Biol. anim. Bioch. Biophys., 1, 47-69.

Kuzdzal-SAvoIE S., I964. Influence de la composition de la ration sur la composition chimique du beurre de vache. Thèse de docteur en Sciences naturelles. Université de Paris, 147 p.

MatTson S., I961. Lipids in milk. Internat, Dairy Fed. Part II, I-I6.

Moore J. H., Steele W., Noble R. C., I969. The relationships between dietary fatty acids, plasma lipid composition and milk fat secretion in the cow. J. Dairy Res., 36, 383-392.

Noble R. C., Steele W., Moore J. H., I969. The effects of dietary palmitic and stearic acids on milk fat composition in the cow. J. Dairy Res., 36, 375-382.

Richardson T., Mc Gann T. C., 1964. Fatty acids in irish butterfat. Irish J. Agric. Res., 3, 15II57.

Robertson J. A., Hawke J. C., ig64. Studies on rumen metabolism. III. Effect of lipid in vivo and in vitro on microbial activity. J. Sci. Fd A gric., 15, 890-897.

Shorland F. B., Weenink R. O., Johns A. T., I955. Effect of the rumen on dietary fat. Nature, 175, II 29-II3O.

Shorland F. B., Weenink R. O., Johns A. T., et Mc Donald I. R. C., I957. The effect of sheep rumen contents on insaturated fatty acids. Biochem. J., 67, 328-333.

Shorland F. B., I96I. Aceton soluble lipids of grass and other forage plants. II. General observation on the properties of the lipids with special reference to the yield of fatty acids. $J$. Sci. Fd Agric., 12, 39-43.

Stadhouders J., Mulder H., I955. The composition of dutch butterfat. I. Seasonal variations in the insaturated fatty acid composition of butterfat. Neth. Milk Dairy J., 9, I82-I93.

Stadhouders J., Mulder H., r956. The composition of dutch butterfat. II. Seasonal variations in the saturatedfatty acid composition of butterfat. Neth. Milk Dairy J., 10, 53-59.

STEELE W., I969. The effects of dietary palmitic and stearic acids on milk fat composition in the cow. J. Dairy Res., 36, 369-374.

Storry J. E., Tuckey B., Hall A. J., r969. The effect of in travenous infusions of triglycerides on the secretion of milk fat in the cow. Br. J. Nutr., 23, I57-I72.

Storry J. E., Hall A. J., Tuckey B., Millard D., I969. The effects of intravenous infusions of cod-iver and soya-bean oils on the secretion of milk fat in the cow. Br. J. Nutr., 23, I73-I80.

Svensen A., Ystgaard L., 1966. Fatty acid composition of Norvegian butterfat. XVII Congr. Intern. Laiterie, I35-I 44 .

Viviani R., Lenaz G., I963. Sintesi di acidi grassi a lunga contena nel rumen di ovino. Bull. Soc. Ital. Biol. Sper., 39, (24 bis), I-4.

WARD P. F. V., SCOTt T. W., DAwSON R. M. C., 1964. The hydrogenation of insatured fatty acid in the ovine digestive tract. Biochem J., 92, 60-68.

Wright D. E., I959 Hydrogenation of lipids by rumen protozoa. Nature, 184, 875. 